\title{
Índice de medicina preventiva. U na medida integradora de la cobertura de los programas preventivos
}

\author{
Roberto Baechler R ${ }^{1}$, Marcela Barra $\mathbf{R}^{2 \mathbf{a}}$, Alex Soto $\mathbf{P}^{3 \mathbf{b}}$. \\ Coverage of preventive health \\ activities in a Chilean region, \\ calculated using the preventive \\ medicine index
}

Background: Preventive activities of the public health system in Chile are not integrated and there is no parameter assessing the whole population that is benefited with these activities. Aim: To develop and implement a mathematical measure of the coverage of preventive health activities, provided to different age groups. Material and methods: Data was gathered from the monthly statistical reports of the women, children, teenager, adult and elderly health programs in 30 communities of the Seventh Chilean Region. The preventive medicine index (PM) was calculated as the ratio between the population that was ascribed to each program and the population that was a potential beneficiary of such program. Results: In the studied region, the global coverage of preventive medicine, calculated using the PMI, increased from 0.229 in 1999 to 0.370 in 2003. This represents a 61\% increment. However, there are important inequalities in the access to preventive health in the different communities of the region. Conclusions: The PMI revealed a substantial increment in preventive health activities in the studied region (Rev Méd Chile 2007; 135: 777-82).

(Key w ords: Delivery of health care; Health services accessibility; Preventive medicine)

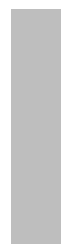

\footnotetext{
Recibido el 16 de agosto, 2006. Aceptado el 3 de octubre, 2006.

${ }^{1}$ Departamento de Epidemiología e Información para la Gestión, Servicio de Salud del Maule, Facultad de Ciencias de la Salud Universidad Católica del Maule. ${ }^{2}$ Departamento de Salud Comunal Talca, Región del Maule. ${ }^{3}$ Departamento de Epidemiología e Información para la Gestión Servicio de Salud del Maule.

aMatrona, Magíster en Salud Pública

bLicenciado en Estadística
}

L a Medicina Preventiva es el área de la salud pública que se preocupa de mantener a la población sana y de pesquisar precozmente a las

Correspondencia a: Roberto Baechler R. 1 Oriente 940, Talca. Fono: (71) 206652 Fax: (71) 206653.

E mail: rbaechler@ssmaule.cl personas enfermas asintomáticas, su aporte está fundamentado en la posibilidad de intervenir sobre la conducta o el estilo de vida de las personas y en detectar en sus fases iniciales problemas de salud de alta prevalencia. Los programas preventivos constituyen intervenciones de alta costo-efectividad, la evidencia científica ha 
confirmado que la implementación de este tipo de prestaciones, favorece mejorías en el nivel de salud poblacional a un bajo costo para la sociedad ${ }^{1-8}$.

El interés por las actividades preventivas, se ha incrementado en los países que tienen como política de salud el uso eficiente de los recursos y la contención de costos. Asimismo, la orientación de los servicios de salud hacia la prevención, es un elemento utilizado por muchos países como estrategia para lograr una mayor equidad social. En Chile, el Ministerio de Salud ha definido como estrategia de desarrollo fortalecer el nivel primario de atención y enfatizar el enfoque preventivo $2,5,9,10,16$.

En el Sistema Nacional de Servicios de Salud de Chile, la cuantificación de la población incorporada a las actividades de medicina preventiva se hace a través de la medición de la cobertura, este concepto señala la proporción de sujetos adscritos a un programa en relación al total de individuos potencialmente beneficiarios. Trimestral o semestralmente, los establecimientos de atención primaria informan al servicio de salud correspondiente, la cobertura observada en cada prestación preventiva en formularios estadísticos especialmente diseñados para estos efectos ${ }^{11-15}$.

En el sistema de salud público chileno, las coberturas de las distintas actividades preventivas son analizadas en forma independiente, no existe una medida que permita visualizar en forma integral, la proporción de la población incorporada a este tipo de prestaciones. El objetivo del presente trabajo es desarrollar (crear) e implementar una medida que integre matemáticamente, la cobertura de las actividades preventivas que se otorgan a los diferentes grupos etáreos en el Sistema Nacional de Servicio de Salud en Chile.

\section{Material y MÉTOdo}

Aspectos conceptuales. El Índice de Medicina Preventiva (IMP) integra en un solo guarismo, la proporción de población incorporada a las actividades de Medicina Preventiva que se otorgan en los establecimientos adscritos al Sistema Nacional de Servicios de Salud de Chile. El parámetro puede ser analizado en los diferentes niveles que es posible identificar en el sistema de salud chileno (centro de salud, comuna, región, país).
Componentes del Índice de Medicina Preventiva. El IMP está integrado por las prestaciones preventivas que se otorgan a las personas en los diferentes programas de salud implementados por el Ministerio de Salud de Chile, estas prestaciones son registradas en el sistema de estadísticas que se utiliza en las instituciones de salud pública del país. En la Tabla 1 se presentan los componentes del IMP.

La cobertura de las distintas prestaciones preventivas se calcula sobre la base de la población beneficiaria del sistema público de salud, para ello se utiliza la siguiente expresión matemática:

Cobertura: $\frac{\text { Población adscrita al Programa }}{\text { Total Población potencialmente beneficiaria de ese programa }}$

Matemáticamente el Índice de Medicina Preventiva se puede expresar

$$
\mathrm{IMP}=\sum_{\mathrm{i}=1}^{\mathrm{n} P} \cdot \frac{\left(\mathrm{X}_{\text {observado }}-\mathrm{X}_{\text {mínimo }}\right)}{\left(\mathrm{X}_{\text {máximo }}-\mathrm{X}_{\text {mínimo }}\right)}
$$

$\mathrm{n}=\quad$ Prestaciones preventivas de los diferentes programas de salud.

$\mathrm{P}=\quad$ Proporción de población adscrita a un programa específico en un centro de salud 0 en un área territorial determinada, respecto del total de la población beneficiaria del Sistema Nacional de Servicios de Salud de ese centro de salud o de esa área territorial.

$\mathrm{X}_{\text {observado }}=$ Proporción de los individuos adscritos a la prestación preventiva, respecto del total de la población potencialmente beneficiaria de ese programa en particular.

$\mathrm{X}_{\text {mínimo }}=$ Menor cobertura teórica (0)

$\mathrm{X}_{\text {máximo }}=$ Mayor cobertura teórica (100)

La proporción o importancia relativa (P) asignada a cada componente del Índice de Medicina Preventiva -control de salud del menor de 6 años, control de salud escolar, control salud del adolescente, control ginecológico preventivo, examen de salud preventivo del adulto, control de salud del adulto mayor- está determinada por el porcentaje que representa el respectivo grupo etáreo, respecto del total de la población beneficiaria del sistema público de salud de la zona territorial o del centro de salud objeto de evaluación. 
Tabla 1. Componentes Índice de M edicina Preventiva (IM P)

\begin{tabular}{|llc|}
\hline Componentes & Indicador & Fuente del dato \\
\hline Control salud menor 6 años & Cobertura & REM 02 \\
Control salud escolar 6-10 años & Cobertura & REM 02 \\
Control de salud adolescente 10-19 años & Cobertura & REM 03 \\
Examen preventivo del adulto 20-64 años & Cobertura & REM 04 \\
Control ginecológico preventivo 25-64 años $(*)$ & Cobertura & REM 01 \\
Control de salud adulto mayor en $>65$ años & Cobertura & REM 05 \\
\hline
\end{tabular}

REM =Registros estadísticos mensuales.

(*) El control ginecológico preventivo se calculó promediando la cobertura del examen de pesquisa precoz del cáncer cérvico uterino (Papanicolaou) y la cobertura del examen físico de mamas que se realiza en los centros de atención primaria.

Normalización de los datos. Para construir el índice cada indicador de cobertura debe ser normalizado, este método permite adimensionar las cifras y construir matemáticamente un índice con variables de distinta naturaleza. El procedimiento hace que cada expresión numérica adquiera un valor que fluctúa entre cero y uno, sin llevar asociada alguna unidad de medida. Para ello se utiliza la siguiente expresión matemática:

Valor observado - Valor mínimo teórico

Valor máximo teórico - Valor mínimo teórico

El significado de la normalización es:

- Numerador. Indica la distancia que existe entre el valor observado en la cobertura de la prestación preventiva de un área geográfica y el valor mínimo teórico.

- Denominador. Determina el rango teórico en el cual se pueden ubicar los datos.

$\mathrm{Si}$ el valor observado es cercano al máximo, la expresión tiende a 1; por el contrario, si la cifra es próxima al mínimo, el guarismo se acerca a cero.

Recolección de la información. Los datos de cada una de las variables incorporadas en el IMP, son consignados en los registros estadísticos mensuales (REM) en cada establecimiento de atención primaria, la información es recopilada y consolidada por la Unidad de Estadística del Servicio de Salud respectivo, trimestral o semestralmente, según corresponda. Para efectos de la construcción del índice que se presenta en este trabajo, se revisaron los formularios estadísticos del Servicio de Salud del Maule correspondientes al período 1999-2003 que se detallan a continuación:

- REM 01 Programa de la Mujer

- REM 02 Programa de Salud Infantil

- REM 03 Programa de Salud del Adolescente

- REM 04 Programa del Adulto

- REM 05 Programa Salud del Adulto Mayor

Medición del Índice de Medicina Preventiva. Se calculó el Índice de Medicina Preventiva para cada una de las 30 comunas de la VII Región para el período en estudio, para ello se realizó el siguiente procedimiento:

a) Se determinó la proporción (P) de población beneficiaria de cada programa preventivo incorporado en el Índice de Medicina Preventiva para cada una de las unidades de análisis, el cálculo se realizó sobre el total de población beneficiaria del sistema público de salud de cada comuna. b) Se determinó la cobertura observada el mes de diciembre de los años 1999 a 2003 de cada prestación preventiva incorporada en el IMP en cada una de las unidades de análisis. c) Se eliminó la unidad de medida de las cifras que componen el Índice de Medicina Preventiva, para ello se utilizó la siguiente expresión:

$$
\frac{X_{\text {observado }}-X_{\text {mínimo }}}{X_{\text {máximo }}-X_{\text {mínimo }}}
$$

El IMP del año 1999 incluye el control de salud del menor de 6 años, el control de salud escolar de los niños entre 6 y 10 años, el control de salud del adolescente del grupo de 10 a 19 años y el 
control ginecológico preventivo de mujeres entre 25 a 64 años, ese año el índice no incorpora el examen de medicina preventiva del adulto y el control de salud del adulto mayor, debido a que estas prestaciones comenzaron a realizarse el año 2000 en la región del Maule.

Para analizar los resultados del IMP en las distintas comunas, se clasificó los logros de cobertura alcanzados en 4 categorías (cuartiles). Los grupos se identificaron con los conceptos de muy desfavorable percentil 25 o menor, desfavorable percentil 26 a 50, favorable percentil 51 a 75 y muy favorable percentil 76 o mayor.

\section{Resultados}

El Índice de Medicina Preventiva de la región del Maule el año 1999 fue 0,229, este valor se incrementó a 0,370 en el año 2003, la variación porcentual observada en el periodo en estudio fue de $61,1 \%$. Si bien, todas las comunas presentaron un aumento de cobertura en sus actividades preventivas durante el quinquenio, la variación registrada es desigual en las distintas unidades de análisis. En las comunas de Vichuquén, Curepto, Chanco y San Javier el IMP aumentó en más de $100 \%$, distinto es lo observado en la comuna de Rauco donde el incremento fue sólo 14,6\%. En la Figura 1 se muestra la evolución del índice de medicina preventiva de la región del Maule para los años 1999-2003 y en la Tabla 2 se presentan los principales estadígrafos de esta variable.

De acuerdo a la clasificación de logros definida en la metodología, las comunas de Cauquenes y Hualañé se ubican en el grupo muy favorable, ya que mantuvieron resultados superiores al percentil 75 durante todo el período estudiado; también califican en esta categoría, las comunas de Rauco, Molina, San Rafael y Villa Alegre, ya que su IMP estuvo sobre en el percentil 75 en a lo menos tres de los cinco años analizados. Las comunas de Curicó, Licantén, Pencahue y Linares se sitúan en la categoría favorable, dado que las mediciones de su IMP, mayoritariamente estuvieron entre el percentil 50 y 75 .

Resultados menos auspiciosos se observa en las comunas de Teno, Talca, Pelarco, Río Claro y Retiro, toda vez que tres o más registros de su IMP estuvieron entre los percentiles 25 y 50, lo cual determina que sean clasificadas en el grupo con desempeño desfavorable. Las comunas de Yerbas Buenas, Sagrada Familia y Maule presentan valores del IMP inferiores al percentil 25 durante todo el período en estudio, lo cual hace que se ubiquen en la categonía muy desfavorable; así mismo, las comunas de Romeral, Empedrado y Longaví tuvieron registros inferiores al percentil 25 en a lo menos tres años del periodo en estudio, lo cual determina que también se las considere en este último grupo. No se observa una tendencia definida en las comunas de Colbún, Pelluhue y Curepto. En la Tabla 3 se presenta el IMP para cada una de las comunas para el período 1999-2003.

\section{DISCUSIÓN Y COMENTARIOS}

El Índice de Medicina Preventiva integra la cobertura de las actividades preventivas implementadas por el Ministerio de Salud en Chile para su

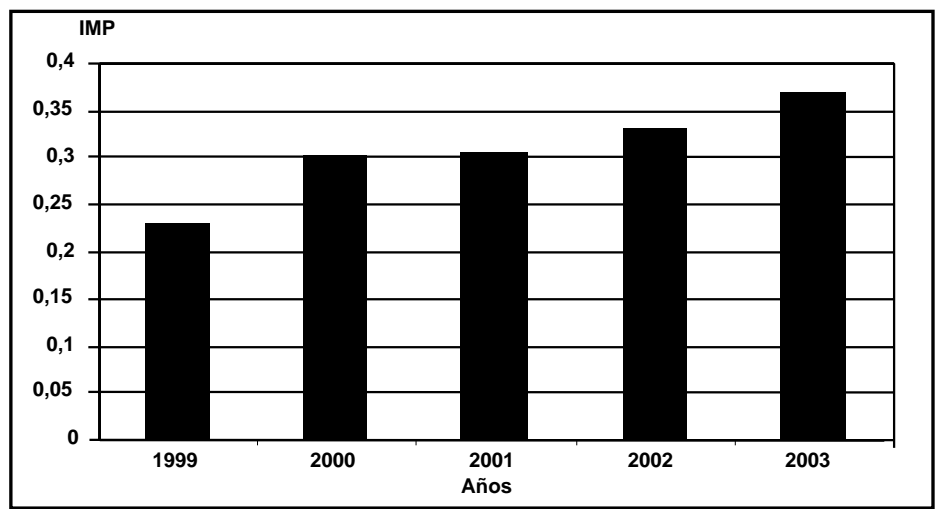

Figura 1. Índice de Medicina Preventiva, Sistema Público de Salud, Región del Maule, años 1999-2003. 
Tabla 2. Principales estadígrafos Índice de M edicina Preventiva VII región 1999-2003

\begin{tabular}{|lccccc|}
\hline & 1999 & 2000 & 2001 & 2002 & 2003 \\
\hline Promedio & 0,231 & 0,295 & 0,298 & 0,344 & 0,387 \\
DesvEst & 0,035 & 0,045 & 0,043 & 0,089 & 0,075 \\
Mediana & 0,229 & 0,288 & 0,288 & 0,328 & 0,380 \\
Cuart25 & 0,210 & 0,267 & 0,271 & 0,291 & 0,336 \\
Cuart75 & 0,245 & 0,313 & 0,319 & 0,369 & 0,416 \\
IMP Max & 0,340 & 0,410 & 0,421 & 0,598 & 0,590 \\
IMP Mín & 0,182 & 0,224 & 0,226 & 0,203 & 0,277 \\
\hline
\end{tabular}

Tabla 3. Índice de M edicina Preventiva Comunas región del M aule 1999-2003

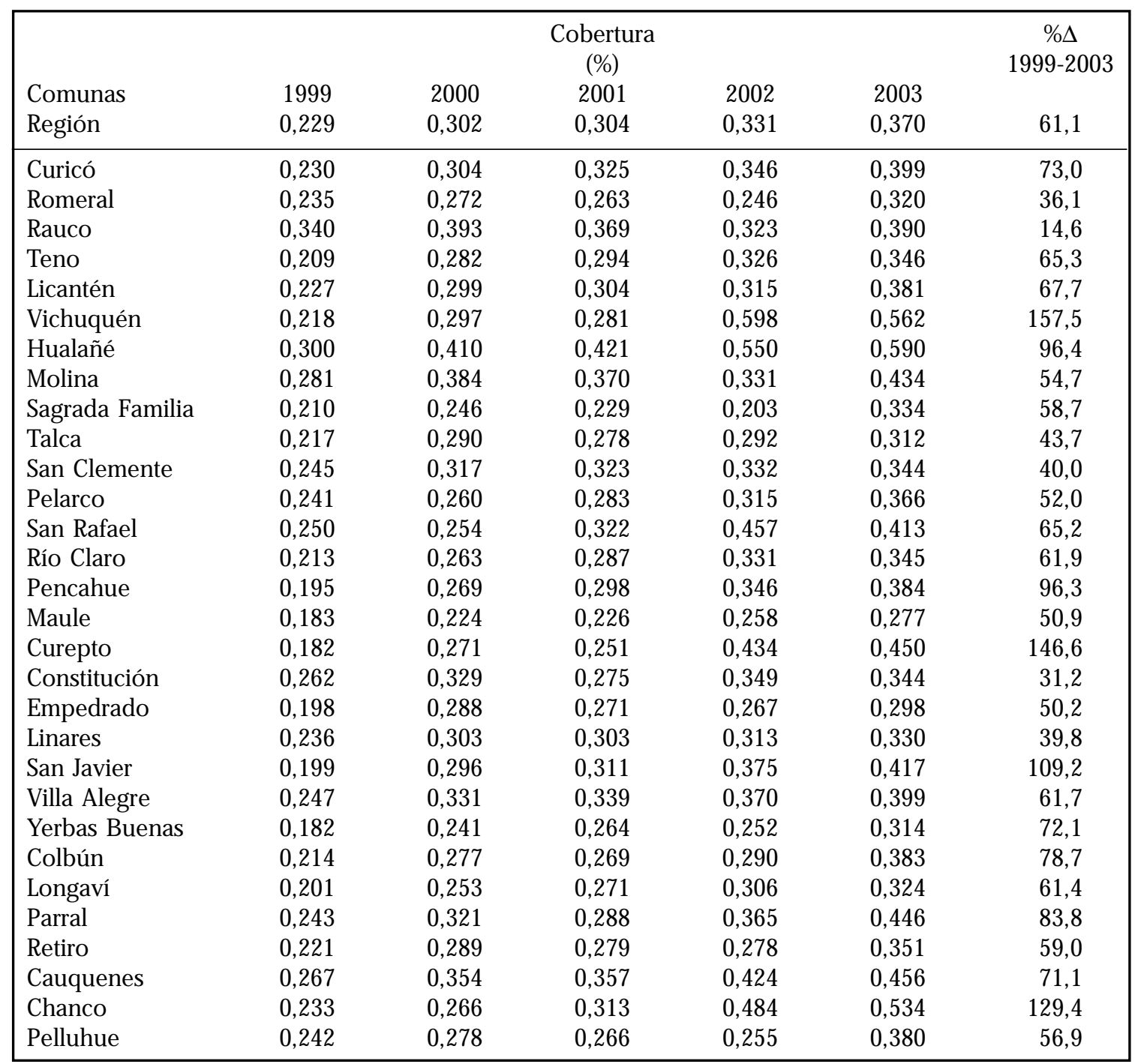


población beneficiaria, el parámetro representa una medida sinóptica de la proporción de personas incorporadas a las prestaciones preventivas en relación a la totalidad de la población potencialmente beneficiaria. En lo conceptual, el IMP establece con claridad el sistema objeto de evaluación, define con precisión sus componentes y todos ellos son coherentes con el sistema representado.

El IMP es mensurable, toda vez que sus componentes están disponibles en los sistemas estadísticos del sistema público de salud; así mismo, es una variable confiable, puesto que mediciones realizadas en similares condiciones reproducen idénticos resultados. Su especificidad permite comparar los resultados de diferentes áreas geográficas. El IMP posee el atributo de sensibilidad, dado que cuantifica todos los cambios de cobertura que se producen en las actividades preventivas en el área estudiada. La integridad del índice, estará determinada por la validez de la información que se registre en los formularios estadísticos, específicamente los datos correspondientes a población bajo control y población beneficiaria del centro de salud o área territorial objeto de análisis. La cuantificación del IMP en la región del Maule durante el quinquenio analizado, muestra que hay un aumento de la población

\section{REFERENCIAS}

1. Guide to clinical preventive services. Second edition. U.S.A. Editorial Williams y Wilkins. 1996.

2. Piedrola Gil. Medicina Preventiva y Salud Pública, novena edición. Barcelona. Editorial Masson Salvat. 2003.

3. JENICEK M. Epidemiología. Barcelona. Editorial Masson. 1996.

4. Martín A, Cano IF. Atención Primaria, tercera edición. Madrid. Editorial Mosby. 1996.

5. Alvarez R. Salud Pública y Medicina Preventiva, segunda edición. México. Editorial Manual Moderno. 1998.

6. GaLo Valiejos FI. Manual del Residente de Medicina Familiar y Comunitaria. Segunda edición. Madrid. Editorial Semfyc. 1997.

7. Citun I, Gómez T. Medicina de Familia la clave de un nuevo modelo. Madrid. Editorial Semfyc. 1997. beneficiaria incorporada a las actividades preventivas. No obstante, los resultados obtenidos el año 2003 están lejos de ser considerados satisfactorios, ya que un IMP de 0,37 indica que $63 \%$ de la población de la VII región todavía no accede a prestaciones preventivas. Además, el estudio deja en evidencia importantes desigualdades en el acceso a actividades de medicina preventiva en las distintas comunas de la VII región.

La aplicación de esta metodología es de alta costo-efectividad, ya que los resultados obtenidos aportan información relevante sobre el acceso de la población beneficiaria a las actividades preventivas a un costo reducido. El IMP puede ser utilizado como una herramienta analítica de apoyo a la gestión, toda vez que permite orientar las intervenciones preventivas hacia aquellos grupos más desprotegidos y; además, permite focalizar el trabajo en las áreas geográficas con menores coberturas.

En resumen, el Índice de Medicina Preventiva entrega una visión sinóptica de la población adscrita a las actividades preventivas desarrolladas en el sistema de salud público de Chile, aportando información epidemiológica de relevancia para los procesos de evaluación del nivel de salud poblacional.

8. Quinta Conferencia Mundial de Promoción de la Salud Ciudad de México. 5-9 Junio 2000. OPS/OMS.

9. Diseño e Implementación de las Prioridades de Salud. La Reforma Programática Chilena. Ministerio de Salud de Chile. 1997.

10. Series de documentos de regulación de normas. Prioridades Salud País. Tomo VII. Ministerio de Salud de Chile. 1998.

11. Programa de Salud del Niño y del Adolescente. Ministerio de Salud de Chile. 1991.

12. Política Nacional de Salud para Adolescentes y Jóvenes. Ministerio de Salud de Chile. 1999.

13. Programa de Salud de la Mujer. Ministerio de Salud de Chile. 1997.

14. Examen Salud Preventivo del Adulto. Ministerio de Salud de Chile. 2003.

15. Norma de la Atención Integral de Salud del Adulto Mayor. Ministerio de Salud de Chile. 2002.

16. Orientaciones para la programación local 2002. Ministerio de Salud de Chile. 2001. 